\title{
BMJ Open Incidence and fatality of serious suicide attempts in a predominantly rural population in Shandong, China: a public health surveillance study
}

\author{
Jiandong Sun, ${ }^{1}$ Xiaolei Guo, ${ }^{2}$ Jiyu Zhang, ${ }^{2}$ Mei Wang, ${ }^{2}$ Cunxian $\mathrm{Jia}^{3}$ Aiqiang $\mathrm{Xu}{ }^{2,3}$
}

To cite: Sun J, Guo X, Zhang J, et al. Incidence and fatality of serious suicide attempts in a predominantly rural population in Shandong, China: a public health surveillance study. BMJ Open 2015;5:e006762.

doi:10.1136/bmjopen-2014006762

- Prepublication history for this paper is available online. To view these files please visit the journal online (http://dx.doi.org/10.1136/ bmjopen-2014-006762).

Received 28 September 2014 Revised 24 November 2014 Accepted 10 December 2014

CrossMark

\begin{abstract}
${ }^{1}$ Queensland University of Technology, Brisbane, Queensland, Australia

${ }^{2}$ Shandong Provincial Centre for Disease Control and

Prevention, Jinan, Shandong, China

${ }^{3}$ Shandong University, Jinan, Shandong, China
\end{abstract}

Correspondence to Professor Aiqiang Xu; aqxuepi@163.com

\section{ABSTRACT}

Objectives: To estimate the incidence of serious suicide attempts (SSAs, defined as suicide attempts resulting in either death or hospitalisation) and to examine factors associated with fatality among these attempters.

Design: A surveillance study of incidence and mortality. Linked data from two public health surveillance systems were analysed.

Setting: Three selected counties in Shandong, China. Participants: All residents in the three selected counties.

Outcome measures: Incidence rate (per 100000 person-years) and case fatality rate (\%).

Methods: Records of suicide deaths and hospitalisations that occurred among residents in selected counties during 2009-2011 (5 623323 person-years) were extracted from electronic databases of the Disease Surveillance Points (DSP) system and the Injury Surveillance System (ISS) and were linked by name, sex, residence and time of suicide attempt. A multiple logistic regression model was developed to examine the factors associated with a higher or lower fatality rate.

Results: The incidence of SSAs was estimated to be 46 (95\% Cl 44 to 48) per 100000 person-years, which was 1.5 times higher in rural versus urban areas, slightly higher among females, and increased with age. Among all SSAs, 51\% were hospitalised and survived, $9 \%$ were hospitalised but later died and $40 \%$ died with no hospitalisation. Most suicide deaths $(81 \%)$ were not hospitalised and most hospitalised SSAs (85\%) survived. The fatality rate was $49 \%$ overall, but was significantly higher among attempters living in rural areas, who were male, older, with lower education or with a farming occupation. With regard to the method of suicide, fatality was lowest for non-pesticide poisons (7\%) and highest for hanging $(97 \%)$.

Conclusions: The incidence of serious suicide attempts is substantially higher in rural areas than in urban areas of China. The risk of death is influenced by the attempter's sex, age, education level, occupation, method used and season of year.

\section{Strengths and limitations of this study}

- This is the first attempt to estimate the incidence of serious suicide attempts resulting in death or hospitalisation in a homogeneous Chinese population.

- Data from two independent surveillance systems were linked to enable the examination of factors related to a high fatality after suicide attempt.

- A limitation is that our incidence estimates are likely to be underestimated due to underreporting in both surveillance systems.

\section{INTRODUCTION}

Approximately one in every four suicides worldwide occurs in China. ${ }^{1}$ Despite a rapid decrease in the past few decades, ${ }^{2}$ suicide remains a major public health concern in this population, claiming approximately 13 deaths per 100000 persons each year. ${ }^{3}$ Suicide in China has some important features, such as a relatively high suicide rate in women, which is similar to or even higher than the rate in men, ${ }^{3} 4$ a 3-4 times higher suicide rate in rural areas compared to urban areas, ${ }^{45}$ and a very high rate among the elderly ( $\geq 65$ years), which is approximately five times the rate in the general population. ${ }^{4-6}$ In contrast, for much of the western world, the male-female suicide ratio is typically large (2-4 times), while the difference in suicide rates between urban and rural populations, and between the young and the old, is much less pronounced. ${ }^{7-10}$ Additionally, pesticide self-poisoning has been the leading method for suicide in China but is relatively rare in developed countries. $^{11}$

Suicide acts not only lead to a loss of lives but also place a tremendous burden on hospital resources. In fact, of all the serious suicide attempts (SSAs) that require 
immediate hospital care, only a small fraction of these represent fatalities from suicide, especially in developed countries. ${ }^{12}{ }^{13}$ For example, in the USA, it is estimated that for every suicide death there are 13 emergency department (ED) visits and 4-5 hospitalisations due to non-fatal suicide attempts. ${ }^{12}$ Studies have shown that there are important differences between suicide deaths and hospital-admitted attempters, among whom most will survive. ${ }^{12-15}$ Compared with the former, suicide hospitalisations or ED admissions are generally overrepresented by females, younger people and those who use low-lethality suicide methods, such as medication overdose. ${ }^{12-15}$ Despite these differences, the two groups may share the same underlying causes for suicide action, such as psychiatric and psychological disorders and adverse life events, and therefore should be included in suicide prevention, particularly primary prevention targeting causes or risk factors of diseases.

Although several studies have assessed the characteristics of non-fatal suicide attempts in China, ${ }^{14-16}$ all were hospital based and there have been no data on the incidence of the total SSAs in this population. It is also unclear what factors contribute to a fatal outcome after a suicide attempt. Using surveillance data, this study aimed to estimate the incidence of SSAs, defined as suicide attempts resulting in either death or hospitalisation, and to examine if fatality among these attempters differs by the victim's background characteristics (sex, age, education, occupation and urban/rural residency), season of attempt and method used.

\section{METHODS}

Design

This study was a surveillance study where data from two public health surveillance systems in Shandong were analysed. Shandong is one of the most populous provinces in China with a total population of 95.8 million in 2010. ${ }^{17}$ The Disease Surveillance Points (DSP) system, established in the early 1980s and including 19 countylevel sites in 2011, routinely collects information on deaths that occurred in the catchment population (about 13\% of the provincial population). This system functions as per the national protocol for vital registration. ${ }^{18}$ Briefly, public health workers in township-level hospitals collect all death certificates in the catchment populations from hospital records and/or via interviews with family members. These certificates are then sent to local centers for disease prevention and control (CDCs) where an underlying cause of death is assigned to each case.

The recently developed Shandong Injury Surveillance System (ISS), which currently includes six counties/ cities with a total catchment population of 5.6 million, gathers information on all injury admissions to all local hospitals using a standard form. Reporting and data management for ISS are usually undertaken by the same personnel as for the DSP reporting system. It should be noted that the ISS does not include admissions to hospitals outside the county, but the proportion is considered to be minimal. Province-wide data are managed by the Shandong provincial CDC. Regular training and underreporting surveys are conducted on an annual basis to ensure data quality.

\section{Setting and participants}

Three counties were selected as part of the aforementioned surveillance systems and thus have both mortality and hospitalisation data available. The study population was defined mainly because of data availability and could not be seen as a provincial representative sample. Our sample includes all residents $(\mathrm{n}=2.2$ million) from these sites. The three sites are highly homogeneous in terms of ethnicity (ethnical Han Chinese $>99 \%$ ), rural/ urban composition ( $\sim 75 \%$ rural residents) and social economic level measured by gross domestic product per capita (US\$7000 to US\$12000 in 2010). The study period was defined from 2009 to 2011. For each year and location, we examined data completeness defined as reported all-causes deaths being within $\pm 5 \%$ of official figures from local Statistical Bureaus. Data from one site for 2009 and 2011 were excluded from analysis for incompleteness.

\section{Data preparation and categorisation}

We extracted records of suicide deaths and hospitalisations for suicide attempts in the three selected counties from the respective databases, based on the underlying cause of death or the primary diagnosis, respectively. The 10th revision of the International Classification of Diseases and Related Health Problems (ICD-10: X60X84, Y87.0 $)^{19}$ was used for case ascertainment. All records were carefully checked and duplicate reports of the same attempt were integrated. Those who were present in both databases were linked based on name, sex, residence and time of injury. Official population data from local Statistics Bureaus were used in the calculation of incidence rates.

Repeat suicide attempts by the same person were rare. Only 6 of 2571 persons $(0.2 \%)$ attempted suicide twice during the study period. We used the most recent attempt to calculate incidence and fatality rates.

All deaths and hospitalisations due to suicide were pooled into one data set for all SSAs containing method of suicide, date committed, outcome and demographic variables. Based on the reported methods for attempts, all SSAs were categorised into one of the following groups: self-poisoning with pesticide (ICD codes: X68); self-poisoning with other substances (X60-67 and X69); hanging (X70); drowning (X71); cutting or stabbing (X78); jumping (X80); and all other methods (X72-77, $\mathrm{X} 79, \mathrm{X} 81-84, \mathrm{Y} 87.0)$

Residence type, season and age-group

By convention, residence type was classified as urban (city suburbs) and rural (townships and villages). Season 
was defined as the month in which the suicide action was undertaken: spring (March-May), summer (JuneAugust), autumn (September-November) and winter (December-February). Standard age groups (19 age groups, including $0,1-4,5-9,10-14,15-19,20-24$, 25-29, 30-34, 35-39, 40-44, 45-49, 50-54, 55-59, 60-64, $65-69,70-74,75-79,80-84$ and 85 years and older) were used to calculate age-specific rates, and broader groups $(<35,35-49,50-64$ and 65 years and older) were used in fatality analysis.

\section{Statistical analysis}

All analyses were conducted using the $\mathrm{R}$ software (V.3.0.2). The package 'Epitools' ${ }^{20}$ was employed to calculate incidence rates (per 100000 person-years) and 95\% Poisson exact CIs. Incidence of SSAs for residencespecific and gender-specific subpopulations and incidence of suicide deaths and hospitalisations were also calculated.

Fatality rate (\%) was computed as the proportion of cases that died among all SSAs. Differences in fatality between groups were tested using $\chi^{2}$ tests. Associations between selected factors and fatality were tested using multiple logistic regression and results were expressed as ORs and 95\% CIs. Statistical significance was claimed when $\mathrm{p}<0.05$.

\section{Ethical considerations}

All data were routinely collected via existing surveillance systems. Personal information (such as name and detailed residence) was used only to check and link data. No personal information was used in analysis and reporting.

\section{RESULTS}

\section{Incidence and characteristics of SSAs}

In this Chinese population over the study period (20092011, 5015664 person-years), 2571 persons attempted SSA, resulting in an overall incidence rate of $45.7(95 \%$
CI 44.0 to 47.5) per 100000 person-years. Among these SSAs, $60 \%$ were hospitalised (incidence of SSA hospitalisation: 27.6 hospitalisations per 100000 person-years) and $49 \%$ died (SSA death rate: 22.3 deaths per 100000 person-years). The majority $(85 \%, 1315 / 1553)$ of hospitalised SSAs survived and most deaths $(81 \%, 1018 / 1256)$ were not hospitalised.

There was a greater proportion of hospitalisation after SSA or survival among women in either urban or rural areas, and in urban areas for both men and women. In contrast, the probability of SSA death, especially death without hospitalisation, was much higher in men in either urban or rural areas, and in rural areas for both men and women (table 1 ).

The SSA incidence per 100000 person-years among rural residents (53.6) was 2.5 times the rate among urban residents (21.1). The rural-urban rate ratio was similar in both sexes (2.7 in men and 2.4 in women). In both urban and rural areas, the incidence of total SSAs and hospitalised SAAs was higher than the incidence of SSA deaths, while the incidence of SSA deaths was lower among women compared to men (table 2).

The age of these SSAs ranged from 12 to 100 with a mean of $52.6(\mathrm{SD}=3.9)$ years. As shown in figure 1 , the incidence increased exponentially with age in both urban and rural areas and for both men and women. The rural-urban gap was large at age 20 and above and appeared to be larger among the elderly (figure 1). The rural-urban ratio of SSA incidence among people aged 65 years and older was 3.9 (164.1 vs 42.4 per 100000 person-years).

Self-poisoning was the leading method for these attempts, accounting for $79 \%(2021 / 2571)$ of all SSAs. Hanging $(17 \%, \mathrm{n}=431)$ and all other methods combined (including cutting, drowning, jumping and others, 5\%, $\mathrm{n}=119$ ) were responsible for only one-fifth of all SSAs. Pesticide was the predominant type of poison used to commit suicide, making up $87 \%(\mathrm{n}=1768)$ of all selfpoisoning SSAs and $69 \%$ of all SSAs.

Table 1 Number of serious suicide attempts (SSAs) resulting in either death or hospitalisation by residency (urban/rural) and sex in selected sites of Shandong, China*

\begin{tabular}{|c|c|c|c|c|c|c|}
\hline & All SSAs & $\begin{array}{l}\text { Hospitalised } \\
\text { and survived }\end{array}$ & $\begin{array}{l}\text { Hospitalised } \\
\text { but died }\end{array}$ & $\begin{array}{l}\text { Total SSA } \\
\text { hospitalisations }\end{array}$ & $\begin{array}{l}\text { SSA deaths without } \\
\text { hospitalisation }\end{array}$ & $\begin{array}{l}\text { Total SSA } \\
\text { deaths }\end{array}$ \\
\hline \multicolumn{7}{|l|}{ Urban } \\
\hline Female & $154(100)$ & $104(67.5)$ & $18(11.7)$ & $122(79.2)$ & $32(20.8)$ & $50(32.5)$ \\
\hline Male & $132(100)$ & 69 (52.3) & 17 (12.9) & 86 (65.2) & 46 (34.8) & 63 (47.7) \\
\hline Both sexes & 286 (100) & $173(60.5)$ & 35 (12.2) & $208(72.7)$ & 78 (27.3) & 113 (39.5) \\
\hline \multicolumn{7}{|l|}{ Rural } \\
\hline Female & $1174(100)$ & 637 (54.3) & $100(8.5)$ & 737 (62.8) & 437 (37.2) & $537(45.7)$ \\
\hline Male & $1111(100)$ & 505 (45.5) & $103(9.3)$ & 608 (54.7) & 503 (45.3) & 606 (54.5) \\
\hline Both sexes & 2285 (100) & $1142(50.0)$ & 203 (8.9) & 1345 (58.9) & $940(41.1)$ & 1143 (50.0) \\
\hline \multicolumn{7}{|l|}{ Total } \\
\hline Female & 1328 (100) & 741 (55.8) & 118 (8.9) & $859(64.7)$ & 469 (35.3) & $587(44.2)$ \\
\hline Male & $1243(100)$ & 574 (46.2) & $120(9.7)$ & 694 (55.8) & 549 (44.2) & 669 (53.8) \\
\hline Both sexes & 2571 (100) & 1315 (51.1) & 238 (9.3) & $1553(60.4)$ & 1018 (39.6) & 1256 (48.9) \\
\hline
\end{tabular}




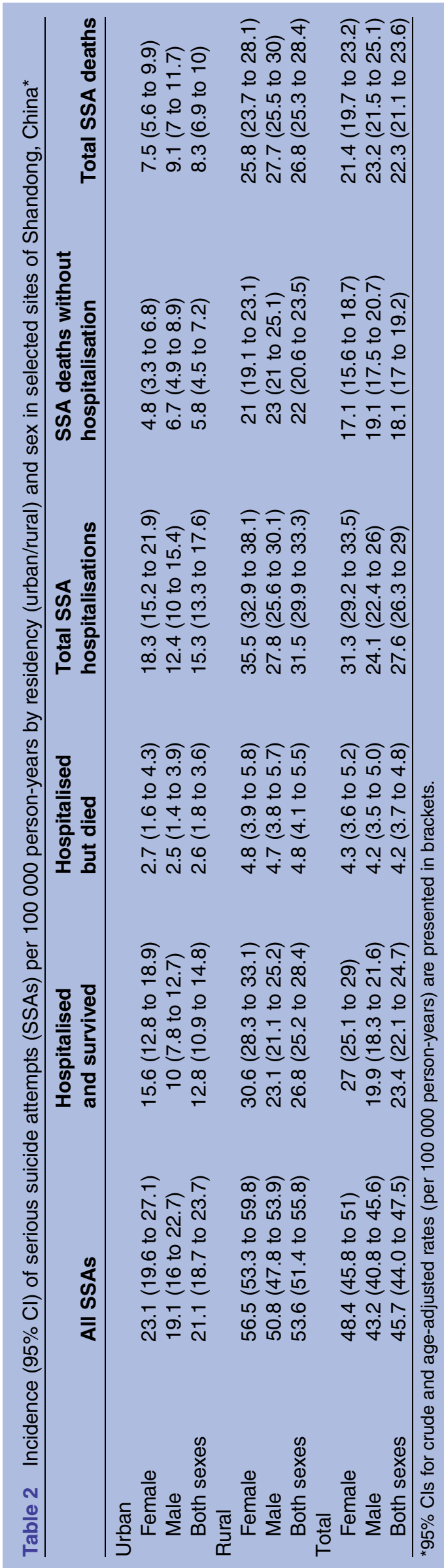

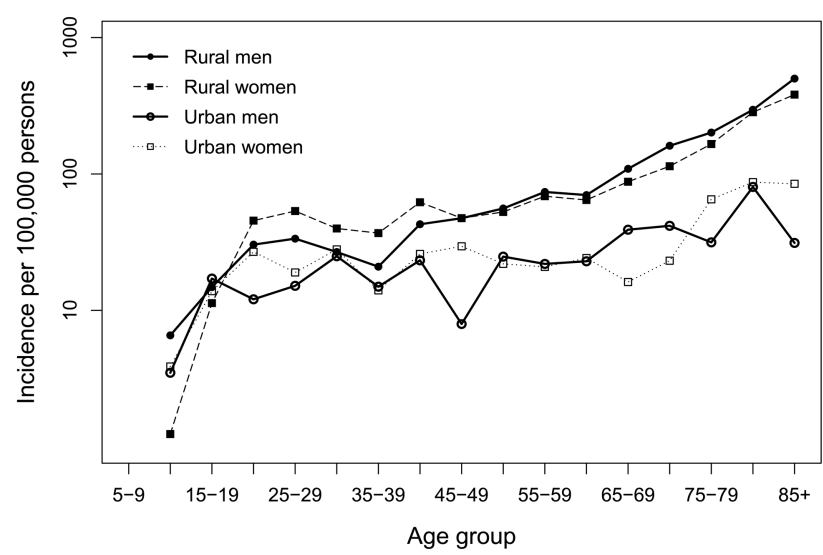

Figure 1 Age-specific incidence rate (per 100000 person-years) at a log scale of serious suicide attempts in a Chinese population in Shandong, China.

\section{Fatality and influencing factors}

As aforementioned, $49 \%$ of all SSAs died. As shown in table 3, the fatality rate was higher among attempters who were from rural areas, who were male, older, with lower education or with a farming occupation (all $\chi^{2}$ tests, $\mathrm{p}<0.001)$. With regard to suicide method, fatality was lowest for other poisons (7\%) and highest for hanging $(97 \%)$. Attempts in non-summer seasons, especially those in spring and winter, had a higher fatality rate than those in summer (table 3 ).

Among those who were hospitalised ( $\mathrm{n}=1553), 1315$ $(85 \%)$ survived and $238(15 \%)$ died. The survival rates for the hospitalised SSAs due to pesticide use, other poisons, hanging and all other methods were $83 \%$ $(998 / 1198), 96 \%(235 / 245), 38 \%(12 / 32)$ and $90 \%$ $(70 / 78)$, respectively. Among those hospitalised SSAs due to self-poisoning using either pesticide or other poisons $(n=1443), 85 \%(1233 / 1443)$ survived.

A multiple logistic regression showed that being male (vs being female: $\mathrm{OR}=1.25,95 \%$ CI 1.01 to 1.55 ), older age (50-64 vs $10-34$ years: $1.54,1.12$ to 2.13 ; 65 years and older vs $10-34$ years: $1.93,1.36$ to 2.75 ), low education (illiterate vs secondary and above: $11.27,7.96$ to 15.96; primary vs secondary and above: $4.99,3.82$ to 6.50 ), farming occupation (vs all other occupations: $1.72,1.29$ to 2.30 ), hanging method (vs pesticide: 29.03 (15.87 to 53.10) and attempting in spring (1.70, 1.28 to $2.25)$ and winter $(1.70,1.27$ to 2.30$)$ versus in summer were significantly associated with a higher fatality rate. Compared with pesticide, attempts with other poisons were associated with a much lower fatality $(0.09,0.05$ to 0.15 ; table 3 ). Residency was no longer a significant factor in this multiple regression model.

\section{DISCUSSION}

To the best of our knowledge, this was the first attempt to estimate the incidence of total serious suicide 
Table 3 Fatality (\% and 95\% Cls) of SSAs and results of multiple logistic regression analysis (ORs and 95\% Cls)

\begin{tabular}{|c|c|c|c|c|c|}
\hline & \multicolumn{3}{|l|}{ Bivariate analysis } & \multicolumn{2}{|c|}{ Multiple logistic regression } \\
\hline & Number of SSAs & Fatality (\%) & p Value ${ }^{\star}$ & OR (95\% Cl) & p Value ${ }^{\star}$ \\
\hline \multicolumn{6}{|l|}{ Residency } \\
\hline Urban & 286 & $113(39.5)$ & \multirow[t]{2}{*}{$<0.001$} & 1.29 (0.93 to 1.81$)$ & NS \\
\hline Rural & 2285 & $1143(50.0)$ & & 1 & Referent \\
\hline \multicolumn{6}{|l|}{ Sex } \\
\hline Female & 1328 & $587(44.2)$ & \multirow[t]{2}{*}{$<0.001$} & 1.00 & Referent \\
\hline Male & 1243 & $669(53.8)$ & & 1.25 (1.01 to 1.55$)$ & 0.036 \\
\hline \multicolumn{6}{|l|}{ Age group } \\
\hline $10-34$ & 575 & $121(21.0)$ & \multirow[t]{4}{*}{$<0.001$} & 1.00 & Referent \\
\hline $35-49$ & 575 & 189 (32.9) & & 1.22 (0.90 to 1.66$)$ & NS \\
\hline $50-64$ & 620 & $343(55.3)$ & & 1.54 (1.12 to 2.13$)$ & 0.008 \\
\hline 65 and above & 801 & $603(75.3)$ & & $1.93(1.36$ to 2.75$)$ & $<0.001$ \\
\hline \multicolumn{6}{|l|}{ Education } \\
\hline Illiterate & 539 & $457(84.8)$ & \multirow[t]{3}{*}{$<0.001$} & 11.27 (7.96 to 15.96$)$ & $<0.001$ \\
\hline Primary & 673 & $472(70.1)$ & & 4.99 (3.82 to 6.50$)$ & $<0.001$ \\
\hline Secondary and above & 1359 & $327(24.1)$ & & 1.00 & Referent \\
\hline \multicolumn{6}{|l|}{ Occupation } \\
\hline Farming & 2032 & $1093(53.8)$ & \multirow[t]{2}{*}{$<0.001$} & 1.72 (1.29 to 2.30$)$ & $<0.001$ \\
\hline All others & 539 & 163 (30.2) & & 1.00 & Referent \\
\hline \multicolumn{6}{|l|}{ Method } \\
\hline Pesticide & 1768 & $770(43.6)$ & \multirow[t]{4}{*}{$<0.001$} & 1.00 & Referent \\
\hline Other poison & 253 & $18(7.1)$ & & 0.09 (0.05 to 0.15$)$ & $<0.001$ \\
\hline Hanging & 431 & $419(97.2)$ & & 29.03 (15.87 to 53.10$)$ & $<0.001$ \\
\hline All others & 119 & $49(41.2)$ & & $1.02(0.66$ to 1.60$)$ & \\
\hline \multicolumn{6}{|l|}{ Season } \\
\hline Summer & 760 & $321(42.2)$ & \multirow[t]{4}{*}{$<0.001$} & 1.00 & Referent \\
\hline Autumn & 605 & $293(48.4)$ & & 1.22 (0.92 to 1.62$)$ & NS \\
\hline Winter & 545 & $286(52.5)$ & & $1.70(1.27$ to 2.30$)$ & $<0.001$ \\
\hline Spring & 661 & 356 (53.9) & & 1.70 (1.28 to 2.25$)$ & $<0.001$ \\
\hline
\end{tabular}

attempts in a Chinese population. We estimated that about 46 (95\% CI 44 to 48) people in every 100000 persons each year had at least one serious suicide attempt that led to either death or hospitalisation. Among them, 51\% were hospitalised and survived, 9\% were hospitalised but died later, and $40 \%$ died with no hospitalisation. Such findings, which are not covered by mortality data, may provide additional information for suicide prevention, particularly primary prevention.

It is surprising to see that the incidence of SSA hospitalisation (27.6 per 100000 persons) was only slightly higher than the suicide death rate (22.3 per 100000 persons). In western populations, however, the hospitalisation-death rate ratio is usually $4-10$ times. $^{12}{ }^{13}$ It is most likely that hospital admission criteria may differ substantially across countries as a function of availability of and access to hospital resources. Many survived attempts in our population, which would have been hospitalised in a western setting, may receive no hospital treatment or only outpatient care, and thus were not included in our SSA hospitalisations. Nevertheless, our SSA population represents the most severe end of the wide spectrum of consequences of suicide acts and should be considered as an important target for suicide prevention.
The rural/urban difference in SSA rates was large (rate ratio=2.5:1) but smaller than the rate ratio for suicide deaths (3.2:1). Additionally, the fatality rate was higher among rural versus urban attempters $(50 \%$ vs $40 \%$ ). These findings may provide some explanation for the large rural-urban gap in suicide rates frequently observed in the national and provincial data in China, ${ }^{4}{ }^{21}$ as the high suicide rate in the rural population is mainly determined by a much higher incidence of serious attempts and, to a lesser extent, a higher fatality rate among rural attempters. However, residence was no longer a significant factor in the multiple regression model (table 3), suggesting that the rural-urban difference in the fatality rate may be explained by the differences in age, education level, occupation and method preference between rural and urban attempters. Nevertheless, these findings emphasise that both primary (to reduce attempts) and secondary (to decrease fatality among attempters) suicide prevention should have a special focus on rural communities in China.

In many western countries, the male-female rate ratio for completed suicide is about $2-4: 1{ }^{7-10}$ however, the incidence of hospitalisations due to suicide is about 1.5 
times higher among females, ${ }^{13} 22$ indicating a much higher fatality rate among male attempters. In this study, we also found a higher rate of SSA hospitalisation (1.3 times the male rate) and a lower rate of suicide mortality in females (male-female ratio 1.1, table 2). These data show, both in our population and in the West, that the higher suicide rate among men is mainly due to a much higher fatality rate among male attempters rather than to a higher incidence of men attempting suicide. Indeed, we found that there was a $25 \%$ (95\% CI 1 to $55 \%$ ) higher odds of death among male versus female attempters (table 3), which was independent of all other factors included in this analysis (table 3). The reasons for this higher fatality rate are unclear but may be associated with residual confounding in suicide method and with other unmeasured factors. For example, although our logistic regression model is adjusted for suicide method, we only have broad method categories and it is unknown whether there is a gender difference in severity when the method category is the same.

We found that most $(79 \%)$ SSAs were due to selfpoisoning and the majority $(85 \%)$ of hospitalised SSAs survived. This indicates a great potential of secondary prevention, that is, early detection and adequate hospital care, to reduce the number of suicide deaths. Although deaths may occur immediately following some suicide acts such as firearm use, for many attempts, especially in China where firearm use is extremely rare and over half of the suicide deaths are due to self-poisoning, ${ }^{5}$ there is a window of time for secondary prevention. In our sample, 578 (46\% of all suicide deaths) self-poisoning SSAs died with no hospitalisation, very likely due to a late detection or referral to a hospital. If these cases can be detected and treated early and a similar survival rate applies, the total number of suicide deaths will reduce by $39 \%(578 \times 85 \%=491)$. However, these SSAs may differ from those hospitalised SSA cases in toxicityand quantity of poison consumed and demographic characteristics. Further research is required to evaluate the potential effects of secondary intervention on suicide.

We identified some risk factors associated with a higher fatality rate after a serious attempt: being male, older age, lower education, having a farming occupation, hanging method and attempting suicide in cold seasons (spring and winter). The underlying reasons are unclear and may be associated with a stronger determination, poorer general health and poorer access to healthcare. These factors should be considered in secondary prevention. A European study has used a similar design by pooling all deaths and hospitalisations due to suicide to analyse risk factors of suicidal fatality and found a higher fatality rate among male and older attempters and those who used highly lethal methods (such as hanging or jumping from a height). ${ }^{23}$

The choice of method was found to be an important factor determining the outcome of suicide attempts. Fatality ranged widely from $7 \%$ in attempters using poisons other than pesticide, to $97 \%$ in hanging attempters. In many developed countries, self-poisoning is also the number one method for suicide attempts overall, but not for suicide deaths, ${ }^{12}{ }^{13}$ because the substances used (mainly medication overdose) have a very low fatality rate. ${ }^{24}$ In contrast, pesticide use represents the leading method for both hospitalisations $(78 \%)$ and deaths $(61 \%)$ in our population, suggesting a very high priority in suicide prevention. Some evidence suggests that restricting access to lethal suicide methods, including pesticide, is one of the most effective suicide prevention strategies. ${ }^{25}$ In the Chinese context, therefore, proper management of pesticide use at a household level and at a population level, and the use of lowtoxicity in place of high-toxicity pesticides in agriculture, may be an effective tool in suicide prevention.

By definition, the incidence of hospitalised attempts in this analysis was underestimated because our surveillance system does not capture those who are treated in neighbouring hospitals in other counties or cities. However, we consider this missing proportion to be minimal because: (1) owing to the emergent nature, most suicide attempters are sent to the nearest hospital; (2) the emergency system is established at the county level and patients using an ambulance will be distributed within local hospitals and (3) government-subsidised health insurance, which covers most rural residents, limits the patient flow to neighbouring hospitals. In addition, some attempters with relatively milder symptoms have been treated in village or community clinics and thus were not included in our analysis. This may hinder the comparison of our SSA incidence with other populations, especially in developed countries. However, the incidence data may be still comparable with other regions in China with similar infrastructure and access to healthcare. Furthermore, this limitation is unlikely to affect the examination of risk factors of fatality among these attempters.

Another limitation is that we used 'self-inflicted injury or poisoning' to identify suicide hospitalisations, and thus some cases with no intention to die may be included. It is difficult to distinguish 'self-harm' from 'suicide attempt' in clinical practice and this definition has been commonly used in surveillance. ${ }^{12} 13$ Our population is limited to three sites with predominantly rural residents. Therefore, the results should be interpreted with caution. Further research with a larger population with approximately equal sizes of urban and rural residents is needed in order to obtain more accurate estimates. It should also be noted that this study was not a cross-cultural study and thus no formal comparison of suicides between Chinese and Western populations can be made. Furthermore, as limited by the data available, we only included a number of demographic factors (gender, age, rural/urban residency, education and occupation), method and timing of attempt in the fatality analysis. Other important factors, such as reason for suicide, intention to die, mental health status and detailed methods (eg, toxicity and quantity of poison ingested) need to be examined in future studies. Finally, 
our data may suffer from limitations associated with surveillance, such as under-reporting and misclassification. Community-based surveys or follow-up studies may be required to further validate findings from this analysis.

In conclusion, this study estimated, for the first time, the incidence of serious suicide attempts in a Chinese population and fatality among these attempters. The incidence of SSAs is much higher in rural areas than in urban areas and slightly higher among females compared to males. It was found that fatality after a serious attempt is markedly influenced by the victim's sex, age, education level, occupation, method used and season of attempt. It is expected that these findings can be used to inform suicide prevention and future research.

Acknowledgements The authors thank all public health workers and professionals who are involved in the Shandong Disease Surveillance Point System and the Shandong Injury Surveillance System. The authors also thank MS Yolanda Lovie-Toon (Queensland University of Technology, Australia) for her assistance in proofreading of the manuscript.

Contributors All authors conceived and designed the study. JS, XG and JZ were responsible for data acquisition and preparation. JS was involved in data analysis. JS, AX, CJ and MW wrote the manuscript.

Funding This research received no specific grant from any funding agency in the public, commercial or not-for-profit sectors

Competing interests None.

Ethics approval Permission to use the data was provided by the data custodian, Shandong CDC. A waiver of full ethics review was obtained from the Shandong CDC Human Research Ethics Committee.

Provenance and peer review Not commissioned; externally peer reviewed.

Data sharing statement Extra data can be accessed via the Dryad data repository at http://datadryad.org/ with the http://dx.doi.org/10.5061/dryad. rov35

Open Access This is an Open Access article distributed in accordance with the Creative Commons Attribution Non Commercial (CC BY-NC 4.0) license, which permits others to distribute, remix, adapt, build upon this work noncommercially, and license their derivative works on different terms, provided the original work is properly cited and the use is non-commercial. See: http:// creativecommons.org/licenses/by-nc/4.0/

\section{REFERENCES}

1. Cui WY. Women and suicide in rural China. Bull World Health Organ 2009;87:888-9.

2. Zhang J, Jing J, Wu X, et al. A sociological analysis of the decline in the suicide rate in china. Social Sci China 2011;5:97-113.
3. Chen Z, Chen Y, Xie Y. Report on the Third National Cause of Death Sampling Survey. Beijing: Peking Union Medical College Press, 2008.

4. Phillips MR, Li XY, Zhang YP. Suicide rates in China, 1995-99. Lancet 2002;359:835-40.

5. Yang GH, Phillips MR, Zhou MG, et al. Understanding the unique characteristics of suicide in China: National psychological autopsy study. Biomed Environ Sci 2005;18:379-89.

6. Li X, Xiao Z, Xiao S. Suicide among the elderly in mainland China. Psychogeriatrics 2009;9:62-6.

7. Hawton K, van Heeringen K. Suicide. Lancet 2009;373:1372-81.

8. Naitonal Institute of Mental Health. Suicide in the U.S. Statistics and Prevention. http://www.nimh.nih.gov/health/publications/ suicide-in-the-us-statistics-and-prevention/index.shtml (accessed 24 Jul 2013).

9. Middleton N, Gunnell D, Frankel S, et al. Urban-rural differences in suicide trends in young adults: England and Wales, 1981-1998. Soc Sci Med 2003;57:1183-94.

10. Yip PSF, Callanan C, Yuen HP. Urban/rural and sex differentials in suicide rates: East and West. J Affect Disord 2000;57:99-106.

11. Ajdacic-Gross V, Weiss MG, Ring M, et al. Methods of suicide: international suicide patterns derived from the WHO mortality database. Bull World Health Organ 2008;86:726-32.

12. Doshi A, Boudreaux ED, Wang N, et al. National study of US emergency department visits for attempted suicide and self-inflicted injury, 1997-2001. Ann Emerg Med 2005;46:369-75.

13. Steenkamp M, Harrison JE. Suicide and hospitalised self-harm in Australia. Canberra, Australia: Australian Institute of Health and Welfare, 2000.

14. Phillips M, Yang G. Suicide and attempted suicide in China, 19902002. MMWR Morb Mortal Wkly Rep 2004;53:481-4.

15. Zhao P, Yang R, Phillips MR. Age-specific characteristics of serious suicide attempters in China. Suicide Life Threat Behav 2010;40:383-93.

16. Guo S. Epidemiological analysis of 674 inpatients with suicide. Chin $J$ Dis Control Prev 2002;6:189.

17. Shandong Office for the 6th Census. Shandong primary data report of the 2010 6th Census (5 May 2011). http://www.stats-sd.gov.cn/ disp/tigb.asp?aa=4220100001 (accessed 14 Jun 2013).

18. Yang G, Hu J, Rao K, et al. Mortality registration and surveillance in China: history, current situation and challenges. Popul Health Metr 2005;3:3.

19. WHO. ICD 10: International Statistical Classification of Diseases and Related Health Problems (Tenth Revisions). Geneva: World Health Organization, 1994.

20. Aragon TJ, Fay MP, Wollschlaeger D. EpiTools: $R$ Package for Epidemiologic Data and Graphics. 2012.

21. Sun J, Guo X, Ma J, et al. Seasonality of suicide in Shandong China, 1991-2009: associations with gender, age, area and methods of suicide. J Affect Disord 2011;135:258-66.

22. Iribarren C, Sidney S, Jacobs DR Jr, et al Hospitalization for suicide attempt and completed suicide: epidemiological features in a managed care population. Soc Psychiatry Psychiatr Epidemiol 2000;35:288-96.

23. Jansen E, Buster MCA, Zuur AL, et al. Fatality of suicide attempts in Amsterdam 1996-2005. Crisis 2009;30:180-5.

24. Eddleston M, Gunnell D. Why suicide rates are high in China Science 2006;311:1711-13.

25. Mann JJ, Apter A, Bertolote J, et al. Suicide prevention strategies. JAMA 2005;294:2064-74. 